\title{
One-Year Serial Follow-up Magnetic Resonance Imaging Study of RigidFix for Femoral Fixation in Anterior Cruciate Ligament Reconstruction
}

\author{
Jung Ho Noh, MD and Jae Woo Lee, MD \\ Department of Orthopaedic Surgery, Kangwon National University Hospital, Kangwon National University School of Medicine, Chuncheon, Korea
}

\begin{abstract}
Purpose: To assess graft slippage at the site of femoral fixation by follow-up magnetic resonance imaging (MRI) after anterior cruciate ligament (ACL) reconstruction with a four-strand autogenous hamstring tendon using RigidFix (DePuy Mitek).

Materials and Methods: Twenty-one subjects diagnosed with ACL rupture underwent ACL reconstruction using hamstring autograft with the transtibial technique. RigidFix was used for femoral fixation and Intrafix and washer screw were used for tibial fixation. Follow-up MRIs were taken immediately after surgery and at 1, 3, 6, and 12 months after surgery. Slippage of the graft and breakage of the RigidFix cross-pin were assessed. Sideto-side difference (SSD) on stress radiographs was measured to assess residual laxity.

Results: Mean slippage of the graft was $0.21 \pm 0.31 \mathrm{~mm}$ at one year after surgery. Cross-pin breakage was noted in two subjects: in one subject, immediately after surgery and in the other subject, at 6 months after surgery. Mean SSD was $1.32 \pm 1.07 \mathrm{~mm}$ at the last follow-up. The correlation coefficient between graft slippage and SSD was $0.131(\mathrm{p}=0.571)$.

Conclusions: RigidFix is a stable fixation device for hamstring autograft with minimally statistically significant but clinically insignificant graft slippage. Cross-pin breakage did not affect the clinical outcomes. Careful preparation of the graft is needed for performing the technique correctly.
\end{abstract}

Keywords: Knee, Anterior cruciate ligament reconstruction, Magnetic resonance imaging, RigidFix

\section{Introduction}

Autogenous hamstring tendon is one of the most popular grafts for primary anterior cruciate ligament (ACL) reconstruction with the development of fixation devices as it causes lower donor site morbidity than autogenous bone-patellar tendon-bone graft ${ }^{1-3)}$. Fixation devices for hamstring tendon graft include post, direct

Received December 9, 2016; Revised (1st) February 4, 2017;

(2nd) February 23, 2017; (3rd) March 3, 2017; (4th) April 15, 2017;

(5th) June 2, 2017; Accepted June 9, 2017

Correspondence to: Jung Ho Noh, MD

Department of Orthopaedic Surgery, Kangwon National University School of Medicine, 1 Gangwondaehak-gil, Chuncheon 24341, Korea

Tel: +82-33-258-9213, Fax: +82-33-258-2149

E-mail: bestknee@hotmail.com

Source of funding: This research was supported by 2016 Research Grant from Kangwon National University.

This is an Open Access article distributed under the terms of the Creative Commons Attribution Non-Commercial License (http://creativecommons.org/licenses/by-nc/4.0/) which permits unrestricted non-commercial use, distribution, and reproduction in any medium, provided the original work is properly cited. cortical fixation, interference screw, button suspension, and cross-pin suspension. RigidFix (DePuy Mitek, Raynham, MA, USA) is composed of two cross-pins that increase the interface area between the graft and the walls of the tunnel. Many reports have shown good results of RigidFix ${ }^{4,5}$. However, there are some potential issues such as graft slippage and pin breakage that should be considered in such a fixation mechanism ${ }^{6,7}$. The purpose of this study is to assess graft slippage at the site of femoral fixation by follow-up magnetic resonance imaging (MRI) after ACL reconstruction with a four-strand autogenous hamstring tendon using RigidFix for at least one year. The null hypothesis is that slippage of the graft at femoral fixation with RigidFix is not significant.

\section{Materials and Methods}

A prospective study was performed in 22 subjects who underwent ACL reconstruction using an autogenous hamstring tendon between July 2008 and December 2011 in National Police Hos- 
pital. The subjects were not selected consecutively. All subjects were informed of the study procedure, the purpose of the study, and any known risks, and they provided informed consent.

Rupture of the ACL was diagnosed by physical examination and 1.5 Tesla MRI (Signa; GE Healthcare, Milwaukee, WI, USA), and it was confirmed by arthroscopy. MRI images were constructed from $2.5 \mathrm{~mm}$-thick slices with a $1 \mathrm{~mm}$ gap between slices, and they had a $256 \times 256$ matrix. On physical examination, Lachman test results was defined as negative (hard end point; side-to-side difference [SSD] $<3 \mathrm{~mm}$ ), $1+$ (SSD of 3-5 mm), 2+ (SSD of 5-10 $\mathrm{mm})$, and $3+(\mathrm{SSD}>10 \mathrm{~mm})$. In addition, a pivot shift test results was defined as negative, $1+$ (glide), $2+$ (clunk), and 3+ (gross). The inclusion criteria were those who underwent primary ACL reconstruction using RigidFix for femoral fixation. Those who had a concurrent medial collateral ligament (MCL) injury that was managed with conservative treatment or a concurrent meniscus tear that was managed with partial meniscectomy were included. The exclusion criteria were as follows: those who had instability of the contralateral knee, multiple ligament injuries of grade 2 or 3 except for MCL injury as the MCL injury can be conservatively managed without alteration of rehabilitation program, grade 3 or 4 cartilage damage requiring additional chondroplasty involving the ipsilateral knee, those who underwent subtotal or total meniscectomy which might affect anterior tibial stability ${ }^{8,9)}$, meniscal repair or meniscal transplantation due to a meniscus injury on which accelerated rehabilitation program cannot be applied, or those who underwent a revision surgery. Prior to surgery, the Telos stress device (Austin \& Associate Inc., Fallston, MD, USA) was used for stress radiography with the knee joint flexed at an angle of $20^{\circ}$ and a force of $150 \mathrm{~N}$ was applied. On stress radiographs, SSD was defined as a difference of anterior translation of the medial compartment between the injured and uninjured knees. The anterior tibial translation was measured for each knee relative to the line tangent to the medial tibial plateau, using the bony landmarks described by Jacob$\operatorname{sen}^{10,11)}$ (Fig. 1). Lachman test and pivot shift test were performed under anesthesia.

\section{Surgical Procedure}

All the operations were performed by a single surgeon (NJH). In all cases, semitendinosus and gracilis tendons were harvested from the ipsilateral side and both tendons were folded into four strands. No. 2 Ethibond was used for whipstitches at $25 \mathrm{~mm}$ of each end of the graft, maintaining a $4-5 \mathrm{~mm}$ interval between stitches. The tip of the tibial tunnel guide was placed at the tibial footprint or at $8 \mathrm{~mm}$ anterior to the posterior cruciate ligament and just lateral to the medial tibial spine. The guide pin was inserted at an angle of $45^{\circ}$ to the tibia. Diameters of the tibial and femoral tunnels were the same as that of the graft. A dilator was not used. The femoral tunnel was prepared via the transtibial technique. The femoral tunnel was drilled to a depth of about 30 $\mathrm{mm}$ at the $10: 30$ position in the right knee or the $1: 30$ position in the left knee while preserving a 1.5-2 mm thickness of the posterior cortical bone. The graft was fixed with RigidFix (DePuy Mitek) in the femoral tunnel. According to the instructions of the manufacturer, correct placement of the femoral fixation system was confirmed by viewing the pin crossing the femoral tunnel via arthroscopy. After the femoral end of the graft was fixed with two cross-pins, pretensioning was achieved by conducting 20 full range of motion cycles under maximal manual load. Tibial IntraFix (DePuy Mitek) was inserted between the four tibial limbs to a depth of 30 to $35 \mathrm{~mm}$ with the knee in $20^{\circ}$ of flexion and with the graft tensioned to $20 \mathrm{lbs}$. Post-tie was performed using a washer screw in all cases. The operation was finished after confirming that there was no impingement on the anterior notch, and then Jones dressing and an immobilizer were applied with the knee in full extension.

\section{Postoperative Rehabilitation}

The hemovac drain was removed on the first or second day after surgery. Prior to the initiation of continuous passive motion

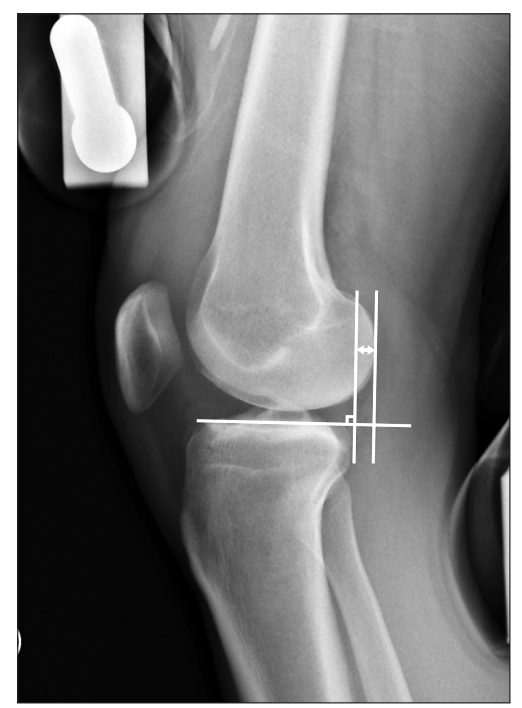

Fig. 1. Anterior tibial translation measurement method. A tangent line to the posterior contour of the medial tibial condyle was drawn perpendicular to the medial tibial plateau. The distance between the line and the posterior edge of the medial femoral condyle was measured (left right arrow). 
(CPM) exercise, postoperative MRI was performed. CPM exercises from $0^{\circ}$ to $50^{\circ}$ began on the fourth postoperative day and advanced to $90^{\circ}$ in the second postoperative week as tolerated. Partial weight bearing was allowed on the fourth postoperative day and total weight bearing as tolerated was permitted. During ambulation, the subjects wore a knee brace locked in extension for a few weeks until quadriceps became stronger. After 12 weeks, jogging and stationary bicycling were allowed. After 6 months, each subject was allowed to participate in competitive sports of one's choice, except for those exercises that might involve strong contact with others or those exercises that might impose strong external forces on the subject. All kinds of exercises were allowed after 9 months. The washer screw was removed at least one year after surgery.

\section{Postoperative Examination}

Lachman test and pivot shift test were performed when the subjects were anesthetized to remove the washer screw. Tegner activity scale was evaluated before injury and at the last followup. In addition to clinical evaluation, follow-up MRIs were performed a few days after surgery, at postoperative 1, 3, and 6 months and one year. Coronal, sagittal, and axial images along the femoral tunnel were added to the routine images (Fig. 2). Stress radiographs were taken using the Telos stress device one year after surgery. MRIs and stress radiographs were measured by two orthopedic surgeons using the picture archiving and communication system (PACS; GE Healthcare, Chicago, IL, USA). To assess slippage of the graft within the femoral tunnel, the distance from the proximal end of the graft to the superior border of the proximal cross-pin was measured on coronal and sagittal images along the femoral tunnel, which was defined as the graft-pin distance (Fig. 3). Measurements on MRI were adjusted based on the known length of the RigidFix cross-pin on the cut image where the entire cross-pin could be seen. The breakage of cross-pin was assessed on coronal and axial images along the femoral tunnel. A positive finding was based on the angulation or a definite gap in either MRI.

\section{Statistical Analysis}

Statistical analysis was performed using SPSS ver. 17.0 (SPSS Inc., Chicago, IL, USA). Correlation between slippage of the graft in the femoral tunnel and residual anterior laxity on stress view was analyzed using a Pearson correlation test. Paired t-test was used for comparison of SSDs before surgery and at the last follow-up, Wilcoxon signed rank test was used for Tegner activity scales before injury and at the last follow-up, and repeated measures analysis of variance (ANOVA) test was used for assessing the graft-pin distances during follow-up. Validation of the repeated measures ANOVA was also performed by Mauchly sphericity test. To test interobserver reliability, the interrater reliability was analyzed using an intraclass correlation coefficient (ICC). The minimum level of statistical significance was $\mathrm{p}=0.05$.

\section{Results}

Twenty one subjects were followed up for at least one year.
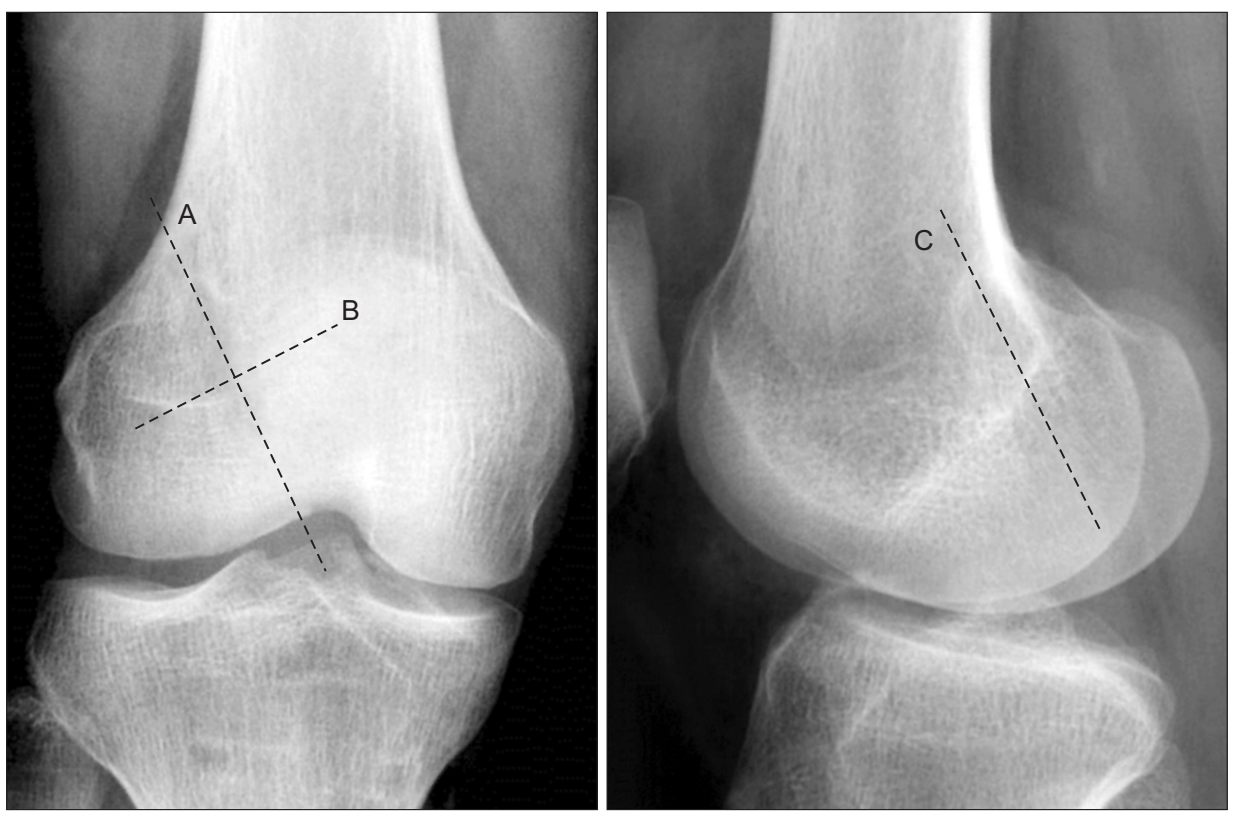

Fig. 2. Sagittal (A), axial (B), and coronal (C) cuts along the femoral tunnel. 

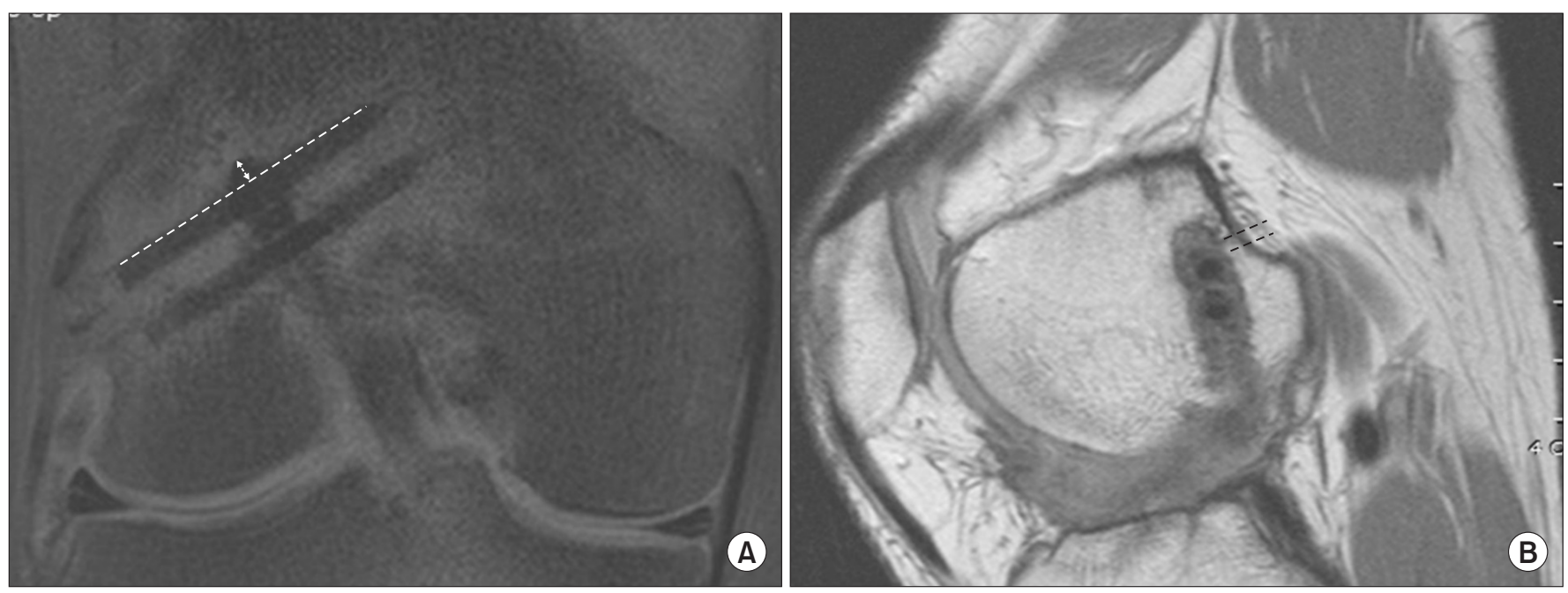

Fig. 3. The graft-pin distance distance was defined as the distance between the proximal end of the graft and the superior border of the proximal cross-pin. (A) Coronal view along the femoral tunnel. Dotted line is the superior border of the proximal cross-pin. Left right arrow is graft-pin distance. (B) Sagittal view along the femoral tunnel. Dotted lines indicate proximal end of the graft and the superior border of the proximal cross-pin.

Their mean age was $26.8 \pm 6.5$ years and mean body mass index was $24.3 \pm 2.0 \mathrm{~kg} / \mathrm{m}^{2}$. Mean period from injury to reconstruction was $13.0 \pm 4.7$ weeks. Two subjects underwent surgeries in acute phase (within 3 weeks after injury) and 12 subjects in chronic phase (more than 3 months after injury). Concurrent injuries were detected in 6 subjects: medial meniscus tear in 2 subjects, lateral meniscus tear in 2 subjects, tears of medial and lateral menisci in 1 subject, and MCL injury in 3 subjects. All subjects with a meniscus tear underwent partial meniscectomy and those with an MCL injury were treated conservatively. Mean followup period was $12.6 \pm 2.5$ months. Mean range of motion was $145.7^{\circ} \pm 5.1^{\circ}$ at the last follow-up. None of the subjects had flexion contracture and one subject had a flexion deficit of $5^{\circ}$ compared to the other knee. Preoperative Lachman test result was $2+$ or $3+$ in all subjects. Preoperative pivot shift test result was negative in 4 subjects, $1+$ in 9 subjects, and $2+$ in 8 subjects. At the last followup, Lachman test result was negative in 19 subjects and $1+$ in 2 subjects. Pivot shift test result was negative in 18 subjects and $1+$ in 3 subjects. All the subjects had removal of washer screw between 12 and 18 months after surgery. Median Tegner activity scale was 6 (range, 5 to 8 ) before injury and 6 (range, 5 to 8 ) at the last follow-up ( $\mathrm{p}=0.046)$. The interobserver ICCs for graftpin distance and SSD were $>0.9$. Mean graft-pin distance was $4.17 \pm 1.05 \mathrm{~mm}$ postoperatively, $4.10 \pm 0.98 \mathrm{~mm}$ at one month, $4.03 \pm 0.97 \mathrm{~mm}$ at three months, $3.98 \pm 0.99 \mathrm{~mm}$ at six months, and $4.00 \pm 1.07 \mathrm{~mm}$ at one year $(\mathrm{p}<0.001)$ (Fig. 4). There was a statistically significant decrease in graft-pin distance between one month and three months in post-hoc analysis $(\mathrm{p}=0.015)$. Mean graft slippage was $0.21 \pm 0.31 \mathrm{~mm}$ at one year after surgery. Mean

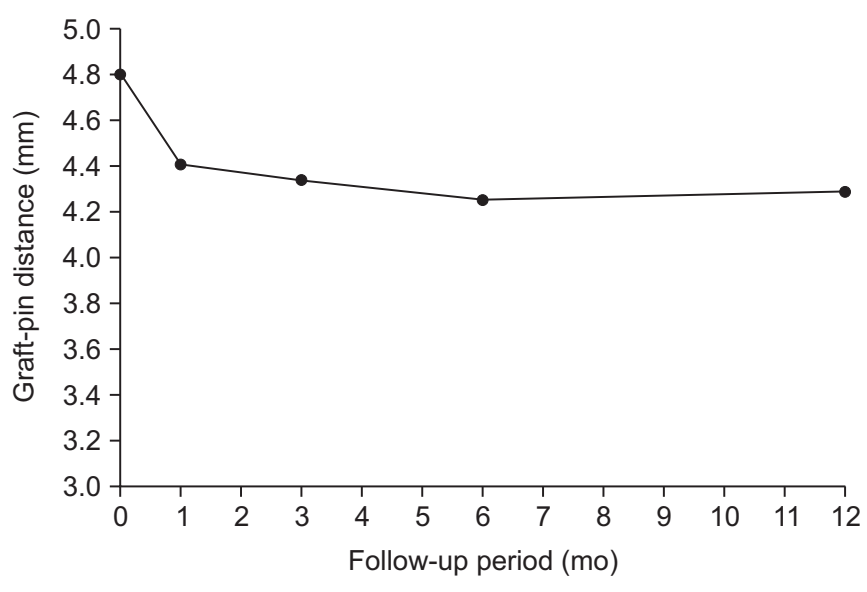

Fig. 4. Graft-pin distance during follow-up.

SSD decreased from $9.10 \pm 2.05 \mathrm{~mm}$ preoperatively to $1.32 \pm 1.07$ $\mathrm{mm}$ at the last follow-up ( $\mathrm{p}<0.001)$. The correlation coefficient between graft slippage and SSD was $0.131(\mathrm{p}=0.571)$. One subject showed breakage of the lower cross-pin on postoperative MRI. In this subject, the cross-pins were in a proper position. The other subject showed breakage of the upper cross-pin on MRI at 6 months after surgery. He did not show breakage of the pin until 3 months after surgery (Fig. 5). At the last follow-up, one subject with early cross-pin breakage showed $1+$ in Lachman test, negative in pivot shift test, and $1.74 \mathrm{~mm}$ of SSD on stress radiography. The other subject with delayed cross-pin breakage showed negative in Lachman test and pivot shift test, and $1.33 \mathrm{~mm}$ of SSD on stress radiography at the last follow-up. No complication was observed during follow-up. 

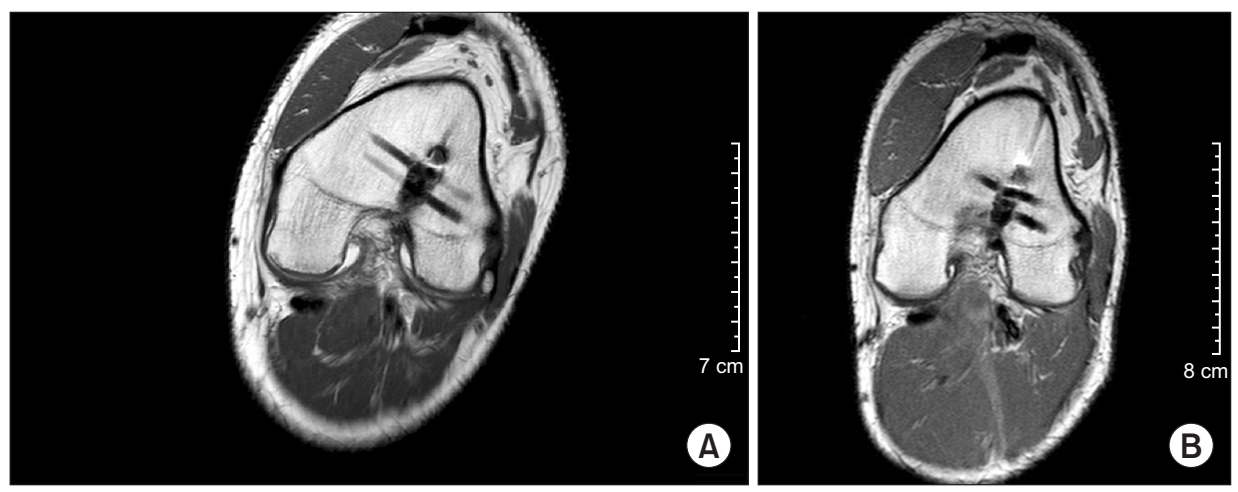

Fig. 5. (A) The upper cross-pin was slightly bent on magnetic resonance imaging (MRI) at 3 months. (B) Breakage of the cross-pin was observed on MRI at 6 months.

\section{Discussion}

This study showed that the graft-pin distance immediately after surgery was different from that at one year after surgery with statistical significance, and based on this finding, we rejected the null hypothesis. However, clinical results including stability were satisfactory at the last follow-up. This study also supports previous studies which have shown that the RigidFix for femoral fixation of the graft was appropriate to obtain satisfactory outcomes $^{12,13)}$. Advantages of using a biomaterial are that there is no need to remove it after the surgery, and if revision surgery is needed, it is relatively easier once the implant has been absorbed. For RigidFix, two bioabsorbable cross-pins with a diameter of 3.3 $\mathrm{mm}$ are passed into the graft in the femoral tunnel. By increasing the volume of the graft in the tunnel, it plays a role in fixing the graft by pressure effect on the wall. Singhatat et al. ${ }^{14)}$ and Zantop et al. ${ }^{15)}$ reported that the failure load and stiffness of RigidFix were excellent compared with those of the interference screw for tendon-to-bone fixation. However, residual laxity might have occurred due to slippage of the graft because cross-pins could not completely fix the tendon graft. Ahmad et al. ${ }^{7)}$ attempted to minimize slippage by engaging cross-pins between the whipstitch sutures placed in the proximal part of the graft, and Dargel et al. ${ }^{16)}$ emphasized the importance of preparation of the graft for reconstruction using RigidFix. According to these authors, the ultimate failure load might be notably decreased if whipstitches are not performed on the graft. RigidFix has been reported to be strong enough to avoid failure and secure enough to resist slippage under cyclic loading during early rehabilitation ${ }^{5)}$. Noyes et al. $^{17)}$ and Morrison ${ }^{18)}$ noted that the degree of intensity which is needed for the ACL would be approximately $450 \mathrm{~N}$ for maintaining the activities of daily living. Kousa et al. ${ }^{19)}$ reported that the failure load was $868 \pm 171 \mathrm{~N}$ on a single-cycle load-to-failure test of RigidFix. Wu et al. ${ }^{20)}$ reported that the ultimate failure load was $676.2 \pm 133.7 \mathrm{~N}$ on a cyclic load test. However, these studies were conducted in an in vitro setting. The manual force of the surgeon in this study was approximately 20-30 lbs, which was notably discrepant from the failure load that has been reported previously. The subject who showed breakage of the lower crosspin on the MRI taken on the fourth day had no additional injury to the reconstructed knee after surgery. Breakage occurring immediately after the surgery is considered to have been developed during pretensioning under the maximal manual load following femoral fixation or during tibial fixation under tension. In the authors' opinion, the cause of cross-pin failure might be the whipstitches used to minimize slippage of the graft. Whipstitch interval without considering the distance between two cross-pins can cause uneven stress on each cross-pin while the graft is being loaded. Especially, the upper pin may be loaded greater than the lower pin when the loop of the graft engages the upper pin before the whipstitch engages the lower pin. A further study is needed to clarify the cause of early breakage. Late failure of the cross-pin developed in one subject. Both cross-pins were intact on postoperative and 1-month MRI, but the upper pin was distally bent slightly on 3-month MRI and it was broken on 6-month MRI. Rodeo et al. ${ }^{21)}$ stated that failure of bone-tendon interface did not occur after twelve weeks of implantation of an autogenous tendon graft in bone tunnel in a canine model. We assume that graft motion in tunnels could persist over three months after surgery which caused late breakage of the cross-pin although graft slippage was not statistically significant. Several authors have suggested that a technical error may increase the incidence of cross-pin breakage ${ }^{22-24)}$. Ahn et al. ${ }^{23)}$ reported that a cross-pin was broken in 25 of 53 knees and that cross-pin breakage was related to posterior transcortical breach. Choi et al. ${ }^{24)}$ reported breakage of cross-pins happened in 12 out of 31 subjects and all of these pins were not in their place. They recommended that the trocar should be placed posterior to the center of the lateral femoral cortex to avoid posterior transcortical breach of the medial part of cross-pins. However, the broken cross-pins in this study were 
positioned properly, which may be a reason of low incidence of cross-pin breakage in this study. Actually, the authors have experienced several cases in which it was difficult to place the crosspins in the correct position. This may be because the trocar was inherently positioned not perpendicular to the femoral cortex and not attached tight, leading to the drill bit being bent while drilling and the drill holes missing the center of the femoral tunnel, and finally, the graft had to be fixed with endobutton or posttied instead of RigidFix. It is therefore assumed that the location should be correctly determined in initially forming pinholes and it is important to ensure that the sleeve-trocar construct firmly adheres to the cortex while drilling. However, the subjects who developed cross-pin breakage also showed comparable clinical results in this study. Many authors have also stated that cross-pin breakage does not affect clinical outcomes ${ }^{22-25)}$.

Milano et al. ${ }^{26)}$ reported the decreasing effects of pretensioning on loosening in porcine knees. They described the changes in the length after a 1,000 cyclic load following fixation of the graft after a 20 -cycle pretensioning. Graft slippage was minimal with Biotransfix and maximal with Ligament Anchor and RigidFix. However, this study showed no correlation between graft slippage and residual laxity. Although the postoperative graft-pin distance was different from that at the last follow-up with statistical significance, the amount of graft slippage was negligible and the clinical and radiological outcomes were satisfactory.

Limitations of the current study are as follows: 1) Remodeling of the graft in the femoral tunnel was not considered. There are many reports about remodeling of the tendon graft in bone tunnels. However, Hunt et al. ${ }^{27)}$ reported that the tendon graft still remained in the femoral tunnel and they showed a low signal on MRI after 2 years of ACL reconstruction in their animal study. 2) The sample size was too small to assess the correlation between the cross-pin breakage and clinical outcomes. 3) We assessed slippage of the graft based on the change in the distance between the proximal end of the graft and the cross-pin. However, displacement of the cross-pin itself was not considered. 4) Only slippage at the cross-pin in the femoral tunnel was discussed in this study. Actually, slippage at the apertures of the femoral and tibial tunnels and elongation of the graft itself affect the residual laxity. However, this study focused on the assessment of graft fixation using RigidFix.

\section{Conclusions}

RigidFix is a stable fixation device for hamstring autograft with minimally statistically significant but clinically insignificant graft slippage. Cross-pin breakage did not affect the clinical outcomes. Careful preparation of the graft is needed for performing the technique correctly.

\section{Conflict of Interest}

No potential conflict of interest relevant to this article was reported.

\section{References}

1. Middleton KK, Hamilton T, Irrgang JJ, Karlsson J, Harner $\mathrm{CD}, \mathrm{Fu} \mathrm{FH}$. Anatomic anterior cruciate ligament (ACL) reconstruction: a global perspective: part 1. Knee Surg Sports Traumatol Arthrosc. 2014;22:1467-82.

2. Feller JA, Webster KE. A randomized comparison of patellar tendon and hamstring tendon anterior cruciate ligament reconstruction. Am J Sports Med. 2003;31:564-73.

3. Laxdal G, Kartus J, Hansson L, Heidvall M, Ejerhed L, Karlsson J. A prospective randomized comparison of bone-patellar tendon-bone and hamstring grafts for anterior cruciate ligament reconstruction. Arthroscopy. 2005;21:34-42.

4. Ibrahim SA, Abdul Ghafar S, Marwan Y, Mahgoub AM, Al Misfer A, Farouk H, Wagdy M, Alherran H, Khirait S. Intratunnel versus extratunnel autologous hamstring doublebundle graft for anterior cruciate ligament reconstruction: a comparison of 2 femoral fixation procedures. Am J Sports Med. 2015;43:161-8.

5. Choi NH, Yoo SY, Victoroff BN. Tibial tunnel widening after hamstring anterior cruciate ligament reconstructions: comparison between Rigidfix and Bio-TransFix. Knee. 2013;20: 31-5.

6. Lee YS, Ahn JH, Lim HC, Kim JG, Yoo JH, Wang JH, Park JS. Structural change of soft tissue anterior cruciate ligament reconstructions with cross-pin fixation between immediate and postoperative 8 weeks: a study with use of magnetic resonance imaging. Am J Sports Med. 2009;37:285-90.

7. Ahmad CS, Gardner TR, Groh M, Arnouk J, Levine WN. Mechanical properties of soft tissue femoral fixation devices for anterior cruciate ligament reconstruction. Am J Sports Med. 2004;32:635-40.

8. Yammine K. Effect of partial medial meniscectomy on anterior tibial translation in stable knees: a prospective controlled study on 32 patients. BMC Sports Sci Med Rehabil. 2013;5: 17.

9. Wieser K, Betz M, Farshad M, Vich M, Fucentese SF, Meyer 
DC. Experimental loss of menisci, cartilage and subchondral bone gradually increases anteroposterior knee laxity. Knee Surg Sports Traumatol Arthrosc. 2012;20:2104-8.

10. Jacobsen K. Stress radiographical measurement of the anteroposterior, medial and lateral stability of the knee joint. Acta Orthop Scand. 1976;47:335-4.

11. Jacobsen K. Gonylaxometry. Stress radiographic measurement of passive stability in the knee joints of normal subjects and patients with ligament injuries: accuracy and range of application. Acta Orthop Scand Suppl. 1981;194:1-263.

12. Shervegar S, Nagaraj P, Grover A, Dj NG, Ravoof A. Functional outcome following arthroscopic ACL reconstruction with Rigid Fix: a retrospective observational study. Arch Bone Jt Surg. 2015;3:264-8.

13. Seo SS, Kim CW, Nam TS, Choi SY. ACL reconstruction with autologous hamstring tendon: comparison of short term clinical results between Rigid-fix and PINN-ACL Cross Pin. Knee Surg Relat Res. 2011;23:208-12.

14. Singhatat W, Lawhorn KW, Howell SM, Hull ML. How four weeks of implantation affect the strength and stiffness of a tendon graft in a bone tunnel: a study of two fixation devices in an extraarticular model in ovine. Am J Sports Med. 2002; 30:506-13.

15. Zantop T, Ruemmler M, Welbers B, Langer M, Weimann A, Petersen W. Cyclic loading comparison between biodegradable interference screw fixation and biodegradable double cross-pin fixation of human bone-patellar tendon-bone grafts. Arthroscopy. 2005;21:934-41.

16. Dargel J, Schmidt-Wiethoff R, Heck M, Bruggemann GP, Koebke J. Comparison of initial fixation properties of sutured and nonsutured soft tissue anterior cruciate ligament grafts with femoral cross-pin fixation. Arthroscopy. 2008;24: 96-105.

17. Noyes FR, Butler DL, Grood ES, Zernicke RF, Hefzy MS. Biomechanical analysis of human ligament grafts used in knee-ligament repairs and reconstructions. J Bone Joint Surg Am. 1984;66:344-52.

18. Morrison JB. The mechanics of the knee joint in relation to normal walking. J Biomech. 1970;3:51-61.
19. Kousa P, Jarvinen TL, Vihavainen M, Kannus P, Jarvinen M. The fixation strength of six hamstring tendon graft fixation devices in anterior cruciate ligament reconstruction. Part II: tibial site. Am J Sports Med. 2003;31:182-8.

20. Wu JL, Yeh TT, Shen HC, Cheng CK, Lee CH. Mechanical comparison of biodegradable femoral fixation devices for hamstring tendon graft: a biomechanical study in a porcine model. Clin Biomech (Bristol, Avon). 2009;24:435-40.

21. Rodeo SA, Arnoczky SP, Torzilli PA, Hidaka C, Warren RF. Tendon-healing in a bone tunnel: a biomechanical and histological study in the dog. J Bone Joint Surg Am. 1993;75: 1795-803.

22. Lee YS, Ha JK, Kim YJ, Yang SJ, Lee MY, Kim JG. Comparative outcome analysis of malpositioned and properly positioned fixation groups after hamstring autograft ACL reconstruction with femoral cross-pin fixation. Knee. 2011;18:303.

23. Ahn JH, Lee SA, Choi SH, Wang JH, Yoo JC, Lee SS, Chang MJ. Femoral cross-pin breakage and its effects on the results of anterior cruciate ligament reconstruction using a hamstring autograft. Arthroscopy. 2012;28:1826-32.

24. Choi NH, Lee JH, Victoroff BN. Do broken cross-pins compromise stability after anterior cruciate ligament reconstructions with hamstring tendons? Arthroscopy. 2007;23:133440.

25. Studler U, White LM, Naraghi AM, Tomlinson G, Kunz M, Kahn G, Marks P. Anterior cruciate ligament reconstruction by using bioabsorbable femoral cross pins: MR imaging findings at follow-up and comparison with clinical findings. Radiology. 2010;255:108-16.

26. Milano G, Mulas PD, Ziranu F, Piras S, Manunta A, Fabbriciani C. Comparison between different femoral fixation devices for ACL reconstruction with doubled hamstring tendon graft: a biomechanical analysis. Arthroscopy. 2006;22: 660-8.

27. Hunt $P$, Rehm O, Weiler A. Soft tissue graft interference fit fixation: observations on graft insertion site healing and tunnel remodeling 2 years after ACL reconstruction in sheep. Knee Surg Sports Traumatol Arthrosc. 2006;14:1245-51. 